\title{
Epidemiologie, Versorgungsbedarf und Versorgungsvolumina peripherer Venenkrankheiten in Deutschland: Multi-Source Analyse von Primär- und Sekundärdaten
}

\author{
M. Augustin, R. Sommer, N. Zander, K. Hagenström, C. Sorbe, J. Petersen, I. Schäfer
}

Institut für Versorgungsforschung in der Dermatologie und bei Pflegeberufen (IVDP), Universitätsklinikum Hamburg-Eppendorf (UKE)

\section{Schlüsselwörter \\ Phlebologie, Versorgung, Epidemiologie}

\section{Zusammenfassung}

Hintergrund: Venenleiden sind häufig und medizinisch relevant. Fragestellungen: Wie ist die Versorgung der Venenkrankheiten in Deutschland, wie entwickelt sich der Bedarf? Methoden: Multisource-Analyse von Daten der GKV, des statistischen Bundesamtes sowie Primärstudien in Deutschland. Ergebnisse: In Deutschland leiden mind. 22\% der Bevölkerung (18 Mio.) unter behandlungsbedürftigen Venenerkrankungen. Ambulant werden jährlich 4,1 Mio. phlebologische Behandlungen durchgeführt, stationär etwa 125000 Fälle als Hauptdiagnose dokumentiert, davon ca. 88500 mit Varizen der unteren Extremität und ca. 35300 mit (Thrombo-)Phlebitis. Die Anzahl stationärer Behandlungen für Varizen ist in den letzten 10 Jahren um 16\% zurückgegangen, in den letzten 17 Jahren um $47 \%$, die Anzahl ambulanter phlebologischer Behandlungen in den letzten 5 Jahren jährlich um $5-10 \%$ gestiegen. Ambulant hauptversorgende Fachgruppen sind Chirurgen und Dermatologen, stationär versorgende Chirurgie, Dermatologie und Angiologie sowie Venenfachkliniken. Der Versorgungsbedarf wird zukünftig jährlich um 3-8\% steigen. Die Zusatzweiterbildung "Phlebologie" als Teil der qualitätsgesicherten Versorgung wurde in Deutschland 2016 von 3049 Ärzten geführt. Fazit: Periphere Venenerkrankungen weisen einen hohen Versorgungsbedarf auf. Die Phlebologie stellt eine wichtige Säule der spezialisierten Versorgung dar.

\section{Keywords}

Phlebology, health care, epidemiology
Korrespondenzadresse

Prof. Dr. Matthias Augustin

Competenzzentrum Versorgungsforschung in der Der-

matologie (CVderm),

Institut für Versorgungsforschung in der Dermatologie

und bei Pflegeberufen (IVDP),

Universitätsklinikum Hamburg-Eppendorf (UKE)

Martinistraße 52, 20246 Hamburg

Tel. (040) 7410-55428

Fax (040) 7410-55348

E-Mail:m.augustin@uke.de

www.uke.de
Epidemiology, health care needs and care volumes of peripheral venous diseases in Germany: multi-source analysis of primary and secondary data

Phlebologie 2018; 47: 242-251

https://doi.org/10.12687/phleb2440-5-2018

Eingegangen: 09. Juli 2018

Angenommen: 18. Juli 2018

English version available at: www.thieme.de/phlebo

\section{Summary}

Background: Vein diseases are frequent and medically relevant. Objective: To characterize phlebological health care and treatment needs in Germany. Methods: Multisource analysis of data from the sick funds, the Federal Statistical Office and primary studies. Results: In Germany, at least $22 \%$ of the population (18 million) suffer from venous diseases requiring treatment. 4.1 million phlebological ambulant treatments are carried out annually, about 125,000 hospital cases are documented as the main diagnosis, including approx. 88,500 with varicosis and approx. 35,300 with thrombo(phlebitis). The number of inpatient treatments for varicosis has decreased by $16 \%$ in the last 10 years, by $47 \%$ in the last 17 years, and the number of outpatient phlebological treatments has increased by $5-10 \%$ annually in the last 5 years. Main ambulant health care specialists are surgeons and dermatologists, main inpatient departments are surgery, dermatology and angiology as well as professional vein clinics. Health care needs will increase by $3-8 \%$ annually in the future. Being a major part of quality-assured care, 3,049 physicians in 2016 had a certified additional training in phlebology. Conclusion: Peripheral venous diseases require a high level of care in Germany. Phlebology and certified phlebologists are an important pillar of the specialised care needed. 


\section{Hintergrund}

Periphere Venenerkrankungen zählen in Deutschland zu den häufigsten Krankheiten. Ihr Spektrum reicht von leichten distalen Beschwerden bis zu schweren chronischen Wunden und letalen Komplikationen, wie tiefen Venenthrombosen. Die chronische Venenkrankheit (CVD) umfasst das gesamte Spektrum der anatomischen und funktionellen Anomalien des Venensystems der unteren Extremitäten, angefangen von Besenreisern und Teleangiektasien bis hin zu venösen Ulzera (1). Die chronische Veneninsuffizienz (CVI) bezieht sich dagegen auf jene Zustände der Venenerkrankung, die mit klinischen Symptomen wie Schwellungen, Schweregefühl bzw. Hautkomplikationen wie Stauungsekzem, Hyperpigmentierung, Dermatosklerose bis hin zum Ulcus cruris venosum einhergehen.

Zur leitliniengerechten Versorgung der Venenerkrankungen gehört die Primärprävention der Veneninsuffizienz, die Früherkennung von Risikopatienten und Vermeidung von Triggerfaktoren sowie die sachgerechte konservative und/oder operative Versorgung (2). Schwerpunktversorger sind Ärzte mit Zusatzqualifikation in Phlebologie. Für diese wurde 1993 die Zusatzweiterbildung „Phlebologie“ eingeführt (3). Zur Diskussion steht die zukünftige Versorgung der Menschen mit peripheren Venenkrankheiten der unteren Extremität in Deutschland unter Berücksichtigung von Qualität und Bedarfsdeckung. In der Studie geht es auch um die Frage, welche Bedeutung die Zusatzweiterbildung „Phlebologie" für die Versorgung in Deutschland hat.

\section{Fragestellungen}

1. Wie häufig sind Venenkrankheiten der unteren Extremität in Deutschland?

2. Wie häufig werden sie ambulant, wie häufig stationär versorgt?

3. Durch welche Fachabteilungen/-gruppen findet diese Versorgung zu welchem Anteil statt?

4. Wie hoch ist der zukünftige Versorgungsbedarf für periphere Venenkrankheiten?
5. Welche Bedeutung hat die Versorgung speziell für niedergelassene Dermatologen?

\section{Methoden}

\section{Studiendesign}

Versorgungswissenschaftliche Analyse auf der Basis von Primärdaten (populationsbezogene Surveys, Arztsurveys) und Sekundärdaten (GKV, Destatis) mit deskriptivem Ansatz. Eingesetzt wurden Analyseverfahren der klinischen Epidemiologie, Gesundheitsökonomie und Sozialwissenschaften. Die methodische Vorgehensweise dieser Studie orientiert sich an den Verfahrensanweisungen des Institutes für Versorgungsforschung in der Dermatologie und bei Pflegeberufen (IVDP) sowie den methodischen Standards der Versorgungsforschung (4-12). Sachstand der Analyse ist April 2018, Datenstand 2016.

Zielindikation waren im weiteren Sinne alle Erkrankungen des peripheren Venensystems, die in Deutschland durch die ICD-10-Kodierungen I80.-, I82.- und I83.dokumentiert werden (13). Im engeren Sinne bezogen sich die Analysen auf die ICD-10-Diagnosen I80.- „Thrombose, Phlebitis und Thrombophlebitis" sowie I83.- "Varizen der unteren Extremitäten". Hinzuweisen ist allerdings auf fehlende klinische Differenzierungen, die mit dem ICD-Schlüssel nicht ausreichend abbildbar sind. Für die Primärdatenuntersuchungen durch phlebologisch versierte Ärzte wurden erweiterte Diagnoseschlüssel verwendet. Den Auswertungen des DAK-Datensatzes lag die ICD-10 Ziffer I87 zugrunde.

\section{Epidemiologie der peripheren Ve-} nenerkrankungen - Primärdatenanalyse

Die Daten zur Epidemiologie in Deutschland wurden zum einen den publizierten Schriften, zum anderen einer jüngst finalisierten populationsbezogenen Studie entnommen. In letzterer wurden bundesweit 19104 Personen im erwerbsfähigen Alter in 51 Unternehmen verschiedener Branchen von Venenspezialisten auf das Vorliegen chronischer Venenerkrankungen mittels strukturierter Anamnese, klinischem
Befund sowie Doppler-Ultraschalldiagnostik untersucht (14).

\section{Häufigkeit der stationären Fälle}

Diese wurde zunächst aus den bundesweiten Krankenhausdaten des Statistischen Bundesamtes gewonnen (15). Diese Statistik stellt eine Vollstichprobe der stationären Versorgung dar. Die Analyse erfolgte für die Jahre 2007-2016 deskriptiv unter Verwendung der ICD-10-Codes I80.-, I82.und I83.- (im Vergleich auch zum Jahr 2000) a) für die gesamte Diagnosegruppe, b) nach Fachabteilung, c) beide geschlüsselt nach Geschlecht.

Eine Prüfung der Ergebnisse und die Nutzung der Daten für weitere Auswertungen erfolgte aus dem Auswertungssatz einer gesetzlichen Krankenversicherung (DAK) mit ca. 5,9 Mio. Versicherten. Die Daten wurden der Projektanalyse einer 40\% Stichprobe entnommen, welche für die Klärung der Forschungsfragen mehr als hinreichend ist.

\section{Anteil der Fachabteilungen}

Als Fachabteilungsschlüssel wurde der in der Destatis-Krankenhausstatistik eingesetzte Schlüssel verwendet. Die Krankenhausversorgung wurde ausschließlich auf Fallebene analysiert, da diese auch Abrechnungs- und Vergleichsgrundlage ist. In gleicher Weise wurden die Fachabteilungsschlüssel für die Analyse der Sekundärdaten herangezogen.

\section{Ambulante Versorgung}

Die Leistungen in der ambulanten Versorgung wurden anhand der GKV-Daten analysiert. Grundlage waren hier die Fallziehungen wie vorher beschrieben.

Die Leistungskodierungen sind in $\mathbf{\nabla}$ Tabelle 1 dargestellt.

\section{Ambulanter Facharztanteil}

Der Anteil der jeweiligen Facharztgruppen an der Versorgung wurde mit dem ambulanten Facharztschlüssel ermittelt. 
Tab. 1 Verwendete Kodierungen für die Sekundärdatenanalyse (16)

\begin{tabular}{|c|c|c|c|c|c|}
\hline Beschreibung & Anwendung & GOP & $\begin{array}{l}\text { GOP-Be- } \\
\text { schreibung }\end{array}$ & OPS & OPS-Beschreibung \\
\hline \multicolumn{6}{|c|}{ Operative Maßnahmen, Gefäßintervention } \\
\hline \multirow{11}{*}{$\begin{array}{l}\text { Verödung von } \\
\text { Varizen (Sanie- } \\
\text { rung epifaszia- } \\
\text { ler Refluxstre- } \\
\text { cken) }\end{array}$} & Sanierung epifaszialer Refluxstrecken & 30501 & $\begin{array}{l}\text { Verödung von } \\
\text { Varizen }\end{array}$ & $5-385$ & Unterbindung, Exzision und Stripping von Varizen \\
\hline & $\begin{array}{l}\text { Krossektomie und Stripping V. saphena } \\
\text { magna oder V. saphena parva }\end{array}$ & 30501 & $\begin{array}{l}\text { Verödung von } \\
\text { Varizen }\end{array}$ & $\begin{array}{l}5-385.74 \\
\leftrightarrow\end{array}$ & $\begin{array}{l}\text { Unterbindung, Exzision und Stripping von Varizen: } \\
\text { Crossektomie und } \\
\text { Stripping: Vv. saphenae magna et parva }\end{array}$ \\
\hline & Seitenastexhairese & 30501 & $\begin{array}{l}\text { Verödung von } \\
\text { Varizen }\end{array}$ & $5-385.9$ & $\begin{array}{l}\text { Unterbindung, Exzision und Stripping von Varizen: Ex- } \\
\text { hairese (als selbständiger Eingriff) }\end{array}$ \\
\hline & $\begin{array}{l}\text { Schaumsklerosierung Stammvarikosis } \\
\text { oder Seitenäste (Sklerotherapie) }\end{array}$ & 30501 & $\begin{array}{l}\text { Verödung von } \\
\text { Varizen }\end{array}$ & $5-385 . c \leftrightarrow$ & $\begin{array}{l}\text { Unterbindung, Exzision und Stripping von Varizen: En- } \\
\text { doluminale } \\
\text { Rotationsablation mit gleichzeitiger Sklerosierung }\end{array}$ \\
\hline & Radiofrequenzobliteration (RFO) & 30501 & $\begin{array}{l}\text { Verödung von } \\
\text { Varizen }\end{array}$ & $5-385 . b \leftrightarrow$ & $\begin{array}{l}\text { Unterbindung, Exzision und Stripping von Varizen: En- } \\
\text { doluminale } \\
\text { Radiofrequenzablation }\end{array}$ \\
\hline & Endovenöse Lasertherapie (EVLT) & 30501 & $\begin{array}{l}\text { Verödung von } \\
\text { Varizen }\end{array}$ & $\begin{array}{l}5-385 . a 1 \\
\leftrightarrow\end{array}$ & $\begin{array}{l}\text { Unterbindung, Exzision und Stripping von Varizen: La- } \\
\text { sertherapie: Endovenös [EVLT] }\end{array}$ \\
\hline & $\begin{array}{l}\text { Paratibiale Fasziotomie mit (Perforans- } \\
\text { dissektion/ -ligatur) (Paratibiale Fas- } \\
\text { ziektomie (PTF) T3 - Empfehlung C) }\end{array}$ & 30501 & $\begin{array}{l}\text { Verödung von } \\
\text { Varizen }\end{array}$ & $5-385.6 \leftrightarrow$ & $\begin{array}{l}\text { Unterbindung, Exzision und Stripping von Varizen: En- } \\
\text { doskopische } \\
\text { Diszision der Vv. perforantes mit Fasziotomie (als selb- } \\
\text { ständiger Eingriff) }\end{array}$ \\
\hline & $\begin{array}{l}\text { Endoskopische Subfasziale Dissektion } \\
\text { der Perforansvenen (ESDP) }\end{array}$ & 30501 & $\begin{array}{l}\text { Verödung von } \\
\text { Varizen }\end{array}$ & $\begin{array}{l}5-385.5 \leftrightarrow \\
5-385.6 \leftrightarrow\end{array}$ & $\begin{array}{l}\text { Unterbindung, Exzision und Stripping von Varizen: En- } \\
\text { doskopische } \\
\text { Diszision der Vv. perforantes (als selbständiger Eingriff) }\end{array}$ \\
\hline & $\begin{array}{l}\text { Rekonstruktion und Transplantation } \\
\text { von Venenklappen }\end{array}$ & & & $\begin{array}{l}5-383.9 \\
* *\end{array}$ & $\begin{array}{l}\text { Resektion und Ersatz (Interposition) von (Teilen von) } \\
\text { Blutgefäßen: } \\
\text { Tiefe Venen [6. Stelle: } 1-k, x]\end{array}$ \\
\hline & $\begin{array}{l}\text { Shave-Operation und andere lokale } \\
\text { operative Verfahren z.B. Spalthaut- } \\
\text { transplantation }\end{array}$ & & & $5-913$ & Entfernung oberflächlicher Hautschichten \\
\hline & Destruktion von erkranktem Gewebe & & & $5-915$ & Destruktion von erkranktem Gewebe \\
\hline
\end{tabular}

Analyse des aktuellen und zukünftigen Versorgungsbedarfes

Der aktuelle und der zukünftige Versorgungsbedarf wurden auf der Grundlage der Epidemiologie von Venenkrankheiten, der in den Primärdatenerhebungen festge-

stellten Behandlungsbedarfe sowie der prognostizierten demografischen Entwicklung nach dem Modell des IVDP ( $\triangleright$ Abb. 1) ermittelt.

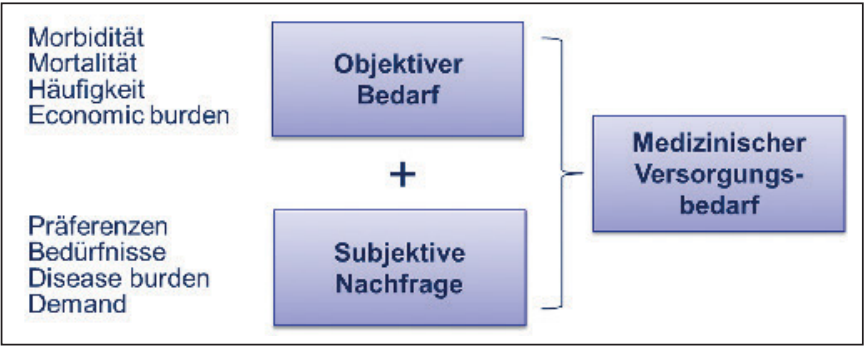

Abb. 1

Systematik der Bedarfsbewertung in der medizinischen Versorgung

\section{Häufigkeit der Zusatzweiter- bildung "Phlebologie"}

Die Häufigkeit der Zusatzweiterbildung "Phlebologie" wurde der Gesundheitsberichterstattung des Bundes entnommen (3).

\section{Ambulante Versorgung durch Dermatologen}

Die Merkmale der dermatologischen phlebologischen Versorgung wurden in einer strukturierten Onlineumfrage 2018 unter niedergelassenen Dermatologen in Deutschland erhoben (17). 
Bedeutung der Zusatzweiterbildung „Phlebologie" für die Versorgung

Die Frage des Anteils und Nutzens von Ärzten mit der Zusatzweiterbildung „Phlebologie“ unter den niedergelassenen Ärzten wurde narrativ in der Gesamtschau der vorliegenden Versorgungsdaten bewertet.

\section{Ergebnisse}

\section{Epidemiologie der peripheren Ve-} nenerkrankungen - Primärdatenanalyse

In der Primärdatenstudie des IVDP wurden 19104 Beschäftigte aus bundesweit 51 Betrieben untersucht. Das Durchschnittsalter betrug 46,1 \pm 9,8 Jahre, 53,5\% waren männlich und der mittlere Body Mass In- dex (BMI) betrug $26,1 \mathrm{~kg} / \mathrm{m}^{2} \pm 4,4$. Von diesen Untersuchten hatten 21,1\% ( $\mathrm{n}=$ 4038) mindestens eine behandlungsbedürftige auf CVI bezogene Diagnose. Eine venenspezifische Behandlung inklusive weiterer Abklärung unklarer Befunde wurde bei $22,3 \%(n=4260)$ empfohlen. Unter Differenzierung nach den CEAP-Kriterien fanden sich bei den Patienten mit Stadien $\geq$ C $1(n=7217)$ in $60 \%$ eine Diagnose mit Varizen $(\triangleright$ Abb. 2)

Die Resultate entsprechen weitgehend den unabhängig zuvor ermittelten Daten von Rabe et al. (18), die bei etwa 17\% der Bevölkerung eine chronische Veneninsuffizienz und damit einen behandlungsbedürftigen Venenbefund der unteren Extremität fanden, ferner eine Gesamtprävalenz von ca. $30 \%$ aller Venenerkrankungen.
Häufigkeit der stationären Fälle 2007-2016

Im Jahr 2016 wurden von Destatis 88561 stationäre Fälle der Diagnose „Varizen der unteren Extremitäten" (ICD-10: I83.-) dokumentiert ( Abb. 3). Von diesen betrafen $51 \%$ Männer und 49\% Frauen. Von diesen gingen ca. 345000 stationäre Behandlungstage aus.

Die Diagnose „Thrombose, Phlebitis und Thrombophlebitis" (ICD-10: I80.-) kam mit 35308 Fällen $(0,18 \%$ aller stationären Fälle in Deutschland) vor. Sie induzierte 321000 stationäre Behandlungstage.

Die Diagnose „Sonstige venöse Embolie und Thrombose" (ICD-10: I82) kam mit 1953 Fällen seltener vor und ist nicht eindeutig einer Lokalisation zuzuordnen. In gleicher Weise sind die unter "Sonstige venöse Embolie und Thrombose" (ICD-10: I87.-) subsummierten Fälle nicht interpretierbar.
Abb. 2

Klinische CEAP-Stadien der Personen mit Venenerkrankungen $(n=7217)(14)$
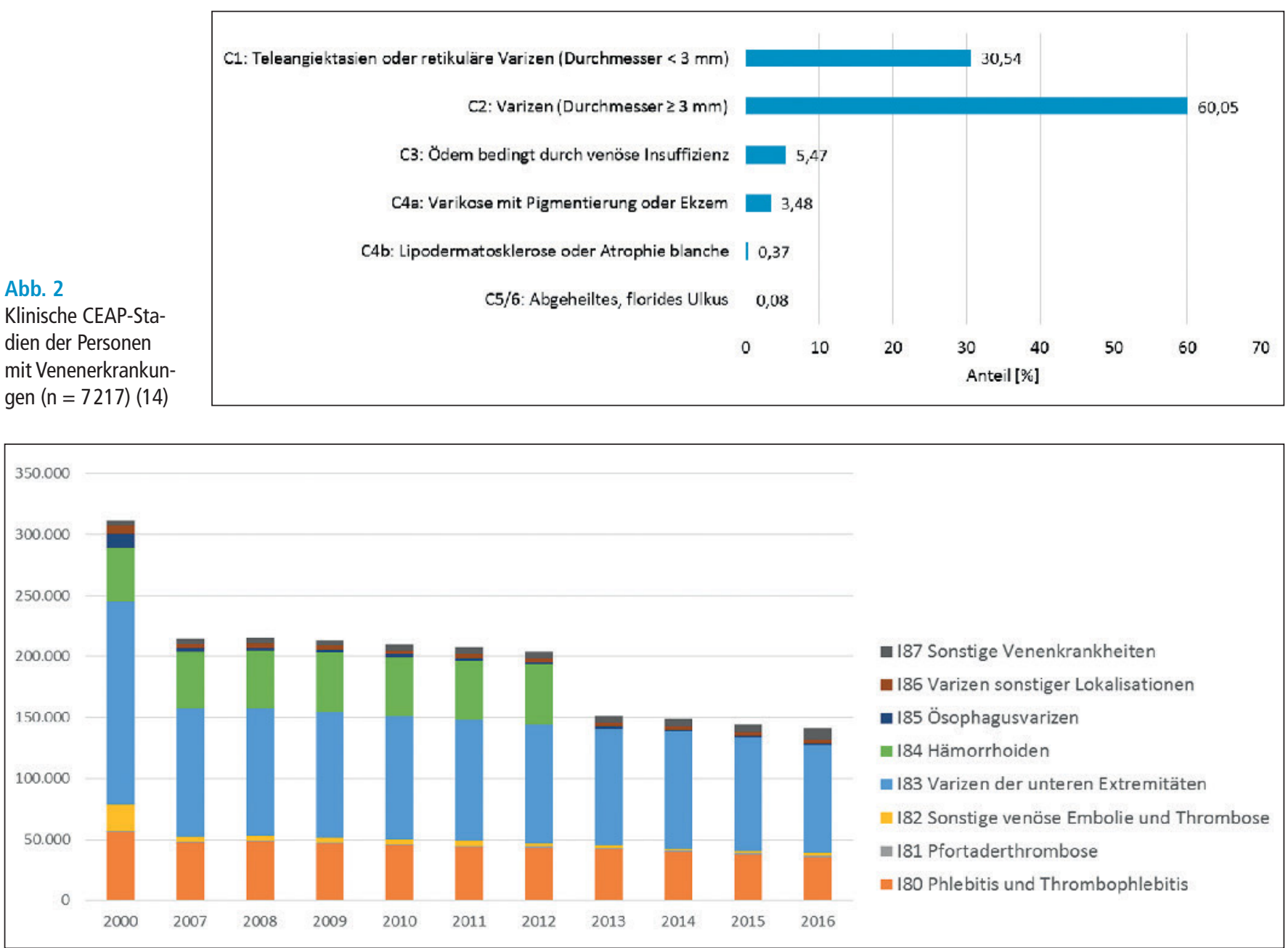

Abb. 3 Stationäre Behandlungstage 2016 für Varizen (ICD-10: I83.-) und andere Venenkrankheiten im Verlaufe der letzten 10 Jahre sowie im Vergleich zu 2000; Daten von Destatis (15) 
Tab. 3 Versorgungsvolumina bei peripheren Venenkrankheiten in den DAK-Daten, Jahr 2010. *IVDP Auswertung DAK Sekundärdaten 2010

\begin{tabular}{|c|c|c|c|c|c|c|c|c|}
\hline & \multicolumn{4}{|c|}{ Rate im Jahr 2010} & \multicolumn{4}{|l|}{ Alter } \\
\hline & $\mathbf{n}$ & Rate (\%) & $\begin{array}{l}\text { unteres } \\
0.95 \mathrm{Cl}(\%)\end{array}$ & $\begin{array}{l}\text { oberes } \\
0.95 \mathrm{Cl}(\%)\end{array}$ & Mittelwert & $\begin{array}{l}\text { Standardab- } \\
\text { weichung }\end{array}$ & Min & Max \\
\hline CVI Total* & 83202 & 3,59 & 3,56 & 3,61 & 67,11 & 14,17 & 3 & 106 \\
\hline CVI-ambulant & 83110 & 3,58 & 3,56 & 3,61 & 67,11 & 14,16 & 3 & 106 \\
\hline CVI-stationär & 168 & 0,01 & 0,01 & 0,01 & 65,29 & 17,52 & 3 & 95 \\
\hline Gefäßinterventionen bei CVI Total * & 3195 & 3,84 & 3,71 & 3,97 & 60,13 & 13,65 & 14 & 96 \\
\hline Gefäß-Intervention-ambulant bei CVI & 2007 & 2,41 & 2,31 & 2,52 & 58,70 & 13,77 & 17 & 96 \\
\hline Gefäß-Intervention-stationär bei CVI & 1274 & 1,53 & 1,45 & 1,61 & 62,40 & 13,01 & 14 & 96 \\
\hline
\end{tabular}

\begin{tabular}{|c|c|c|c|c|}
\hline & \multicolumn{2}{|c|}{ CVI Total* } & \multicolumn{2}{|c|}{$\begin{array}{l}\text { Gefäßinterventionen } \\
\text { bei CVI Total* }\end{array}$} \\
\hline & $\mathbf{n}$ & Rate (\%) & $\mathbf{n}$ & Rate (\%) \\
\hline Baden-Württemberg & 9774 & 3,62 & 494 & 1,34 \\
\hline Bayern & 13407 & 4,04 & 570 & 0,86 \\
\hline Berlin & 2372 & 2,72 & 61 & 0,44 \\
\hline Brandenburg & 2880 & 3,14 & 103 & 0,47 \\
\hline Bremen & 436 & 2,94 & 11 & 0,28 \\
\hline Hamburg & 2254 & 3,14 & 83 & 0,64 \\
\hline Hessen & 7511 & 3,45 & 335 & 1,02 \\
\hline $\begin{array}{l}\text { Mecklenburg-Vorpom- } \\
\text { mern }\end{array}$ & 2484 & 3,80 & 64 & 0,45 \\
\hline Niedersachsen & 7924 & 3,09 & 239 & 0,62 \\
\hline Nordrhein-Westfalen & 16775 & 3,85 & 525 & 0,83 \\
\hline Rheinland-Pfalz & 5352 & 3,76 & 309 & 1,22 \\
\hline Saarland & 1253 & 4,05 & 132 & 2,18 \\
\hline Sachsen & 2136 & 3,43 & 69 & 0,59 \\
\hline Sachsen-Anhalt & 2123 & 4,66 & 41 & 0,40 \\
\hline Schleswig-Holstein & 3039 & 2,68 & 90 & 0,65 \\
\hline Thüringen & 2585 & 4,67 & 37 & 0,24 \\
\hline Keine Zuordnung & 897 & 3,26 & 32 & 0,45 \\
\hline
\end{tabular}

Tab. 2

Anteil von Versicherten mit einer CVI und deren phlebologisch operativ-interventionellen Versorgungsvolumina in den DAK-Daten stratifiziert nach Bundesland, Jahr 2010. *IVDP Auswertung DAK Sekundärdaten 2010

Bei einem Gesamtaufkommen von 20063689 stationären Krankenhausfällen stellen die spezifischen Diagnosen für die Venenkrankheiten der unteren Extremität mit 125822 Fällen einen Anteil von ca. 0,63\% der stationären Behandlungsfälle in Deutschland dar.

Im Vergleich der letzten 10 Jahre lässt sich ein Rückgang der stationären Fälle von 105195 (2007) um 16\% auf 88561 (2016) feststellen. Bei I80.- „Thrombose, Phlebitis und Thrombophlebitis“ lag dieser Rückgang mit 25\% noch etwas höher. Im Ver- gleich zur vorausgehenden Abnahme zwischen 2000 und 2007 hat sich der Rückgang etwas verringert.

\section{Anteil der Fachabteilungen}

Aus der Verbindung von GKV- und Destatis-Krankenhausstatistik geht hervor, dass unter den 88561 stationären Behandlungsfällen die am häufigsten interventionell versorgenden Fachabteilungen die allgemeinchirurgischen mit 32\% und die gefäßchirurgischen mit 23\% waren. An dritter Stelle folgte mit 16\% die Dermatologie, gefolgt von mehreren internistischen Fachabteilungen.

\section{Ambulante Versorgung}

Die Leistungen in der ambulanten Versorgung wurden anhand der GKV-Daten analysiert. Insgesamt wurden im Jahr 2010 unter den DAK-Versicherten 83202 Behandlungen verzeichnet, davon 83110 ambulant und 168 stationär ( Tab. 2). Die JahresBehandlungsprävalenz (ICD-10 GM I87) betrug damit 3,59\%, darunter 3,58\% ambulant und 0,01\% stationär. Pro Jahr lag der Anstieg an Behandlungen bis 2015 zwischen $5 \%$ und $10 \%$.

Insgesamt wurden 3195 Gefäßinterventionen vorgenommen, davon 2007 ambulant $(65,8 \%)$ und 1274 stationär (39,9\%).

Bei der Aufschlüsselung nach Bundesländern fanden sich sowohl für die Anzahl an Behandlungen als auch für die Interventionen regionale Unterschiede ( $>$ Tab. 3 ).

\section{Ambulanter Facharztanteil}

Der Anteil der jeweiligen Facharztgruppen an der interventionellen Versorgung (mindestens ein Kontakt im Jahr) der peripheren Venenerkrankungen war bei den Allgemeinchirurgen $(27,2 \%)$ und den Dermatologen $(25,1 \%)$ am höchsten ( Tab. 4), gefolgt von den Hausärzten (18,6\%).

\section{Disease Burden der Venenkrank- heiten}

Von den Venenerkrankungen geht nach den deutschen Daten stadienabhängig eine zunehmende Einschränkung der Lebensqualität aus (19-22). 
Tab. 4 Anteil der Fachgruppen an der ambulanten Versorgung bei den Versicherten der DAK 2010 (Kontakte $\mathrm{n}=2$ 2007). *IVDP Auswertung DAK Sekundärdaten 2010.

\begin{tabular}{l|l|l|}
\hline & \multicolumn{2}{l}{$\begin{array}{l}\text { Mindestens ein Kontakt } \\
\text { pro Patient und Jahr }\end{array}$} \\
\hline & \multicolumn{2}{|l}{$\begin{array}{l}\text { Ambulante Gefäßinterven- } \\
\text { tionen bei CVI }\end{array}$} \\
\hline & $\mathrm{n}$ & \multicolumn{2}{l}{ Rate (\%) } \\
\hline Missing/Kein & 263 & 13,10 \\
Arztbesuch & & 18,63 \\
\hline Hausarzt & 374 & 0,20 \\
\hline Sonstige & 4 & 27,15 \\
\hline Chirurgie & 545 & 12,95 \\
\hline Gefäßchirurgie & 260 & 0,05 \\
\hline Orthopädie & 1 & 25,06 \\
\hline Hautarzt & 503 & 8,42 \\
\hline Internist & 169 &
\end{tabular}

Die ökonomische Bedeutung der Venenkrankheiten ist aufgrund ihrer Häufigkeit groß. Die in fortgeschrittenen Stadien auftretenden Ulcera crurum stellen dabei die hinsichtlich Krankheitskosten bedeutendste Komplikation dar (23, 24).

\section{Analyse des aktuellen und zukünf- tigen Versorgungsbedarfes}

In der Primärdatenstudie des IVDP 2009 bis 2015 hatten $21,1 \%(\mathrm{n}=4038)$ der Untersuchten mindestens eine CVI-bezogene Diagnose. In der logistischen Regressionsanalyse war neben Alter, Geschlecht und BMI unter anderem das Vorkommen von Varikosis in der Familie ein signifikanter Risikofaktor für CVI. Aus dieser Primärdatenstudie ergab sich bei ca. 22,3\% der Untersuchten aus Sicht des untersuchenden Phlebologen ein Behandlungsbedarf. Dieser bezog sich am häufigsten auf eine Kompressionstherapie, gefolgt von phlebochirurgischen Maßnahmen ( Abb. 4) (14).

Eine Einschränkung der Studie ist der Fokus auf erwachsene Personen unter 70 Jahren, was die Prävalenz in der Bevölkerung unterschätzt, da ältere Menschen regelhaft eine höhere Rate an Venenerkrankungen aufweisen. So betrug die Prävalenz im Alter von über 70 Jahren in den Daten des Barmer Report $2011>70 \%$ ( $>$ Abb. 5) (25). Die altersadjustierte Berechnung er- gab auf dieser Basis einen phlebologischen Versorgungsbedarf von mindestens 30\% der Gesamtbevölkerung.

Vor diesem Hintergrund besteht schon heute ein markant größerer Anteil als 22\% in der Gesamtbevölkerung mit Bedarf nach phlebologischer Versorgung. Für die $\mathrm{Zu}-$ kunft ist zudem aufgrund der demografischen Entwicklung eine weitere Zunahme des Versorgungsbedarfes bei peripheren Venenkrankheiten zu erwarten.

\section{Versorgungsqualität}

$\mathrm{Zu}$ erwarten ist auch ein anhaltend steigender Bedarf nach besser qualifizierter Versorgung, da wichtige Qualitätsindikatoren in Deutschland nicht hinreichend erreicht sind, etwa in der Versorgung venöser Ulzera. So sind nur etwa $40 \%$ der venösen Wunden mit der leitliniengerechten Kompressionstherapie versorgt ( Abb. 6) $(26,27)$.

Die Versorgungsqualität nach Leitlinie gemäß Versorgungsindex ist bei spezialisierten Versorgenden von Wunden höher
( Abb. 7) (28). Wichtigster Prädiktor für eine hohe Versorgungsqualität war die Qualifikation (Spezialisierung) der Versorgenden. Somit muss aus medizinischen wie ökonomischen Gründen gewährleistet sein, dass vaskuläre Wunden unter Hinzuziehung der Experten versorgt werden. Venös bedingte Wunden als häufigste Gruppe der chronischen Wunden der unteren Extremität bedürfen der besonderen phlebologischen Behandlung.

Selbst unter den aktiv versorgenden Ärzten und Pflegenden weist nur ein kleinerer Teil eine hinreichende praktische Kompetenz in der Anlage venöser Kompressionsverbände auf (29). Dies legt nahe, eine konsequente, durch phlebologisch versierte Ärzte initiierte Versorgung durchzuführen, deren Qualität systematisch geprüft wird.

\section{Häufigkeit der Zusatzweiterbil- dung "Phlebologie"}

Nachdem im Jahr 1994175 Ärzte die Zusatzweiterbildung „Phlebologie“ führten,

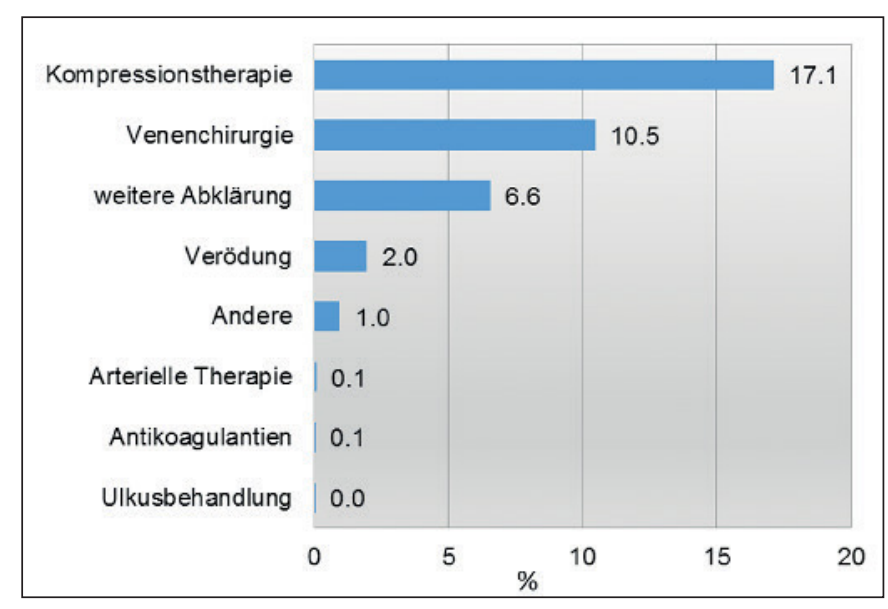

Abb. 4

Notwendige phlebologische Maßnahmen in der werktätigen Allgemeinbevölkerung $(\mathrm{n}=19104)$ (14)

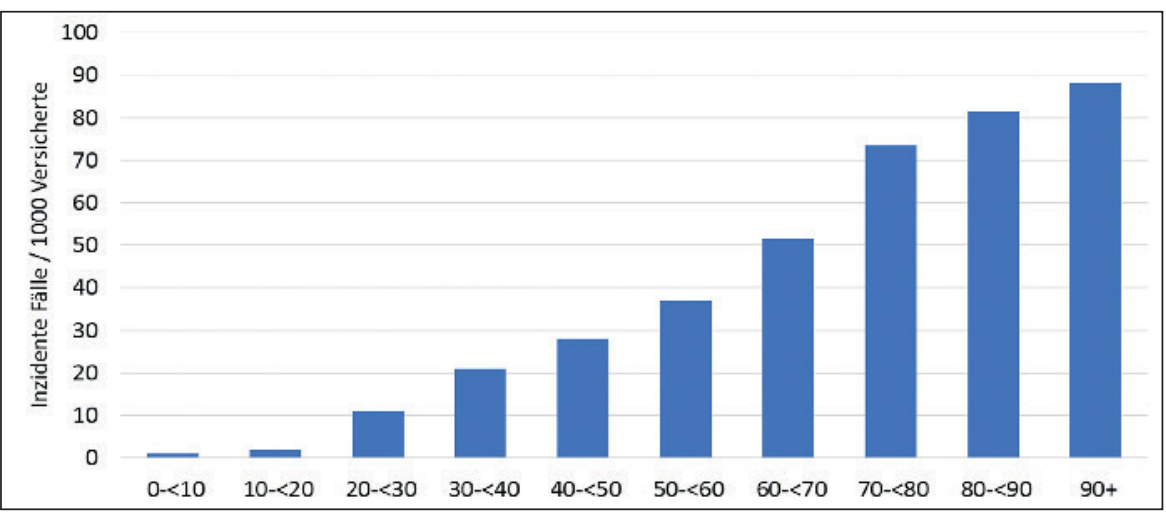

Abb. 5 Altersabhängige Prävalenz von Venenerkrankungen in den Barmer GKV Daten, Report 2011 (25) 


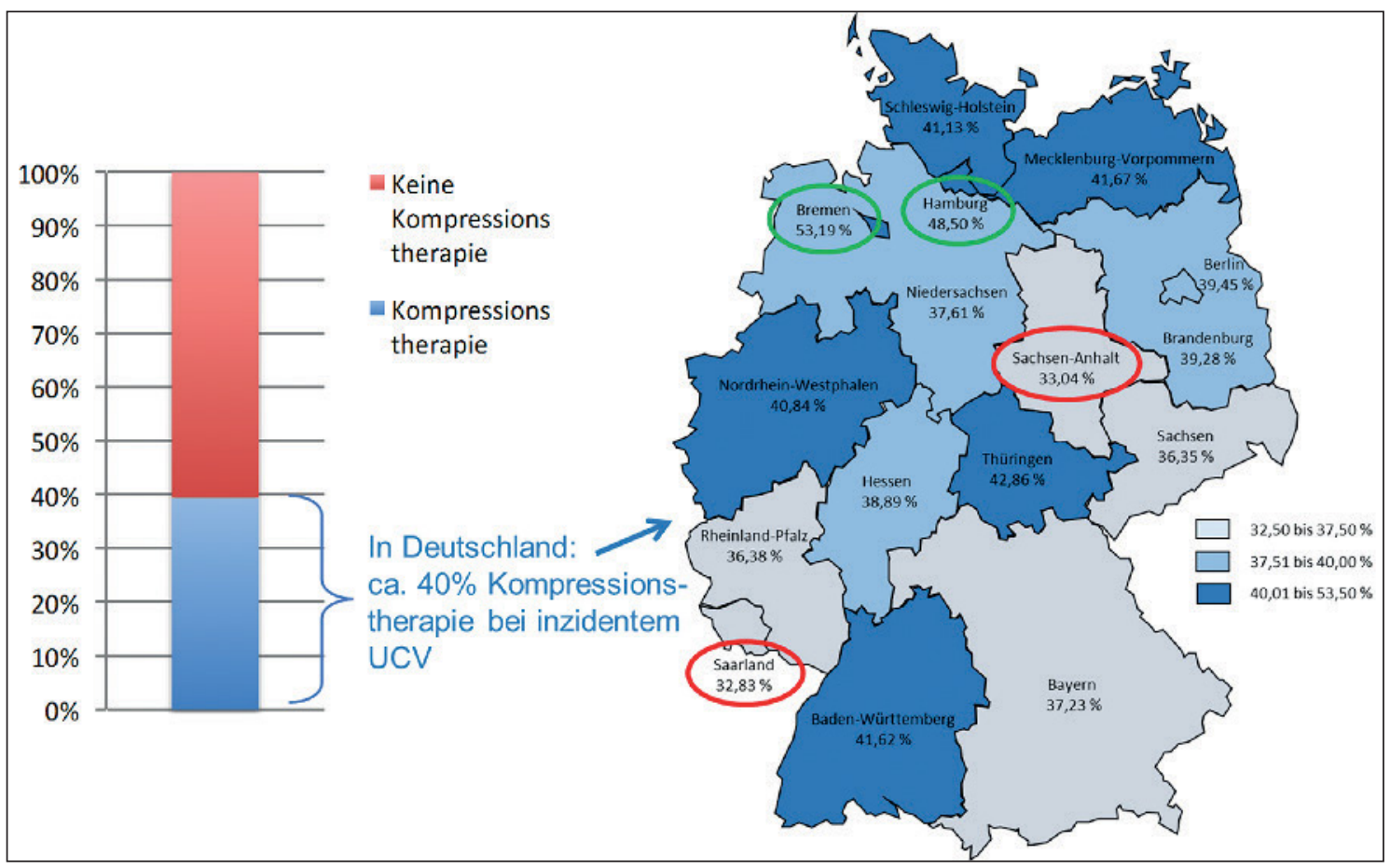

Abb. 6

Versorgung der venösen Ulzera (UCV) in Deutschland mit Kompressionstherapie und deren regionale Unterschiede (Grundlage: $\mathrm{n}=9,1$ Mio Versicherte der Barmer [27])

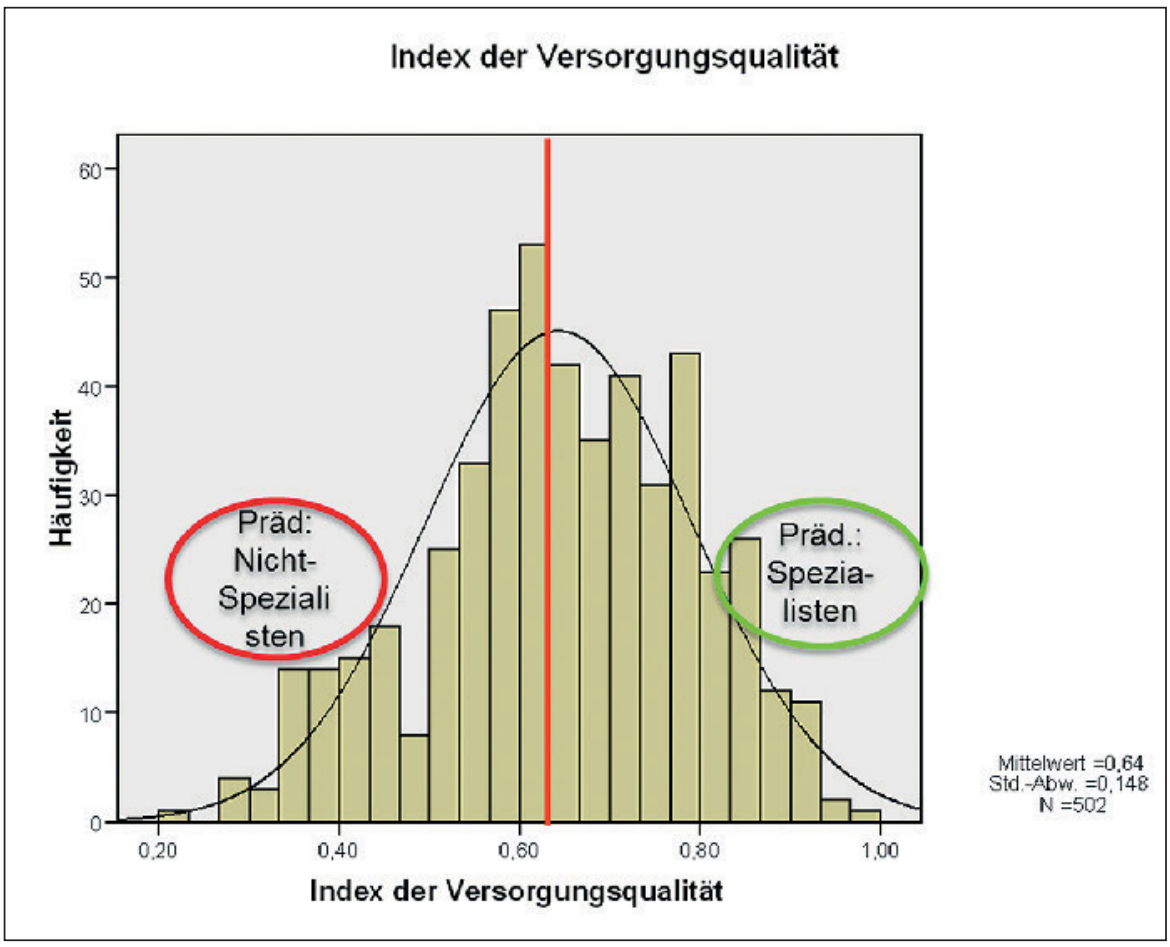

Abb. 7 Verteilung des Versorgungsindex bei chronischen Wunden, inklusive venösen Ulzera (28)

stieg dieser Anteil bis 2016 stetig auf inzwischen 3049 Ärzte ( Abb. 8).

Ziel der vorliegenden versorgungswissenschaftlichen Multisource-Analyse war die Charakterisierung der Versorgung und des
Versorgungsbedarfs von peripheren Venenerkrankungen in Deutschland. In der Gesamtschau ergibt sich aus der hohen Prävalenz von Venenerkrankungen, dem großen Versorgungsbedarf, erhoben durch die Primärdaten, und der hohen Spezialisierung in den einzelnen medizinischen Fachgruppen ein erkennbarer Bedarf an fachtherapeutischer Versorgung der Menschen mit Venenerkrankungen in Deutschland. Für die sachgerechte Versorgung ist auch die Primärprävention sowie die Früherkennung der Risikopatienten und -faktoren von großer Bedeutung. In diesen präventiven Bereichen wie auch in der Kuration müssen evidenzbasiertes, leitliniengerechtes Wissen und manuelle Fertigkeiten in der Breite der Versorgung vorgehalten werden.

Der anhaltende Trend zur Verlagerung der phlebologischen Versorgung in dem ambulanten Bereich ist evident und unterstreicht den wachsenden Versorgungsbedarf in diesem Sektor. In allen Fächern, im Besonderen in der ambulanten Dermatologie und in der Chirurgie, zeigt sich generell eine immer größere Spezialisierung, für die das erworbene Facharztwissen nicht mehr ausreicht. Es ist nicht damit $\mathrm{zu}$ rechnen und kann nicht verantwortet werden, dass die spezialisierte phlebologische Versor- 



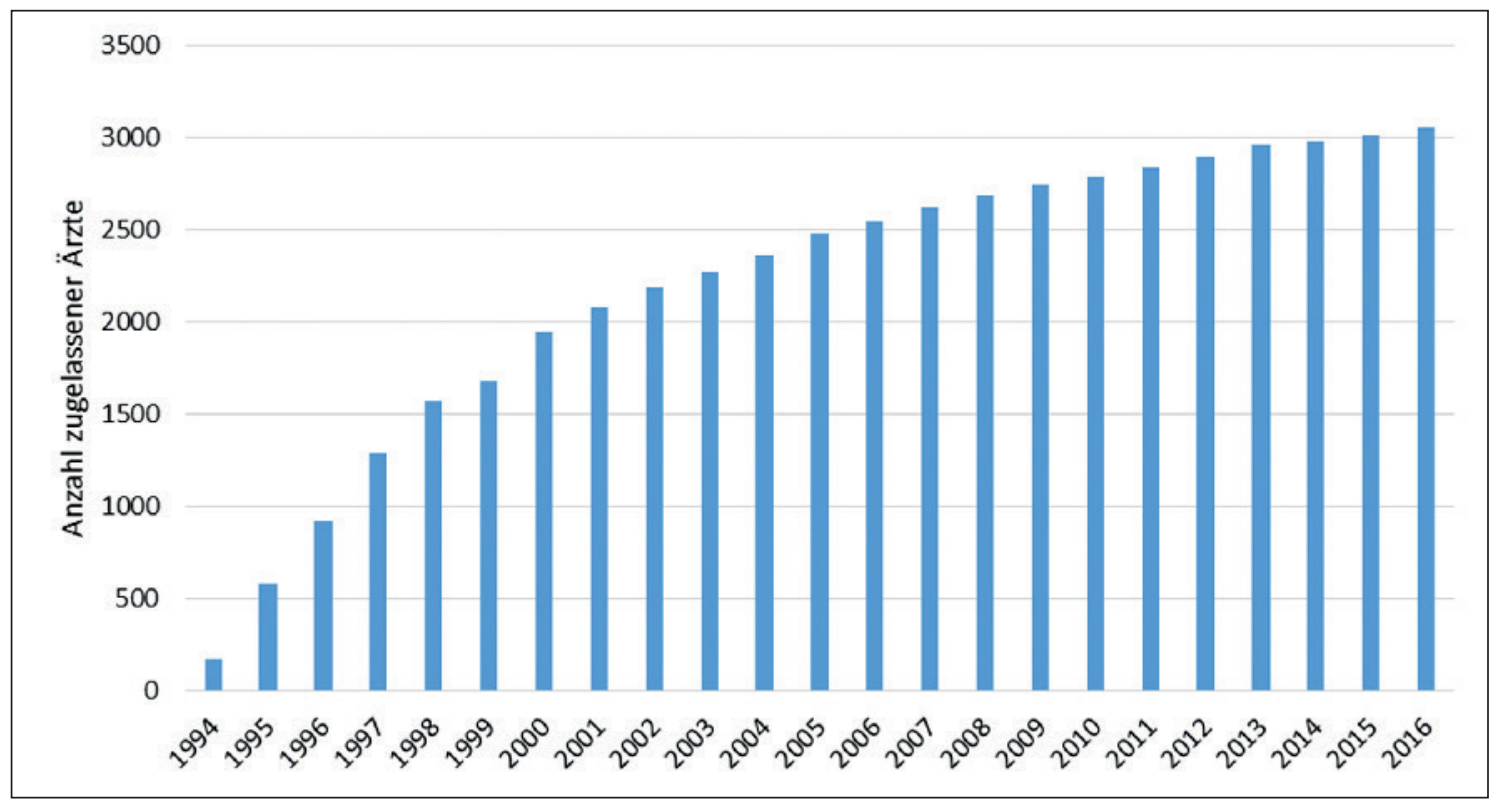

Abb. 8

Anzahl von Ärzten in Deutschland mit der Zusatzweiterbildung „Phlebologie" seit $1994(3)$

gung in der Breite der Fächer geleistet wird. Dagegen sprechen zwei Aspekte:

1) Versorgungsstudien zeigen eindrücklich, dass in Deutschland die fundamentalen Elemente der leitliniengerechten Versorgung von Venenkrankheiten, etwa die Kompressionstherapie bei venöser Insuffizienz, nur von einem kleinen Teil der versorgenden Ärzte und Pflegenden praktisch beherrscht werden (30).

2) Am Qualitätsindikator „Verordnung von Kompressionstherapie “ ist für die venösen Erkrankungen bundesweit eine unzureichende Versorgung festzustellen, dies sowohl in Sekundärdaten (24) wie auch in Primärdaten (8). Zudem weist die sachgerechte phlebologische Versorgung auch signifikante ökonomische Vorteile auf (31). Die Phlebologie ist dabei das breit versorgende, interdisziplinäre Querschnittfach, welches u.a. Allgemeinmediziner, Chirurgen und Dermatologen verbindet. Aus Patientensicht ist insbesondere die Erkennbarkeit der phlebologischen Experten eine unerlässliche sozialrechtliche Grundforderung an das Gesundheitssystem. Die $\mathrm{Zu}$ satzweiterbildung „Phlebologie“ dient dabei auch der optimierten Implementierung von Gesundheitskompetenz in der Bevölkerung, wie sie aus der Breite der Fächer nicht erreicht werden kann.

Insgesamt unterstreicht der vorliegende Multisource-Ansatz auch die hohe Bedeutung der Versorgungsforschung für das
Verständnis, die Qualitätssicherung und zukünftige Ausrichtung wichtiger medizinischer Fächer wie der Phlebologie.

\section{Interessenkonflikt}

Nach Angaben der Autoren bestehen keine Interessenkonflikte.

\section{Ethische Richtlinien}

Für das Manuskript wurden keine Studien an Menschen oder Tieren durchgeführt.

\section{Literatur}

1. Eklöf B, Rutherford RB, Bergan JJ et al. Revision of the CEAP classification for chronic venous disorders: Consensus statement. J Vasc Surg 2004; 40: 1248-1252.

2. Stücker M, Debus ES, Hoffmann J et al. Consensus statement on the symptom-based treatment of chronic venous diseases. J Dtsch Dermatol Ges 2016; 14: 575-83.

3. GBE-Bund. http://www.gbe-bund.de/oowa921-in stall/servlet/oowa/aw92/dboowasys921.xwdevkit/ xwd_init?gbe.isgbetol/xs_start_neu/ \&p_aid=i\&p_aid=33954832\&nummer=612\&p_s prache=D\&p_indsp=93\&p_aid $=42395654$.

4. Glaeske G, Augustin M, Abholz H et al. Epidemiologische Methoden für die Versorgungsforschung. Gesundheitswesen 2009; 71: 685-93.

5. Von Elm E, Altman DG, Egger $M$ et al. The Strengthening the Reporting of Observational Studies in Epidemiology (STROBE) statement: guidelines for reporting observational studies. J Clin Epidemiol 2018; 61: 344-349.
6. Schulenburg JM, Greiner W, Jost F et al. Deutsche Empfehlungen zur gesundheitsökonomischen Evaluation - dritte und aktualisierte Fassung des Hannoveraner Konsens. Gesundh ökon Qual manag 2007; 12: 285-290.

7. Augustin M. Versorgungsforschung bei schweren chronischen Erkrankungen: Ergebnisse und Nutzen am Beispiel chronischer Wunden. In: Trittin C (Hrsg.): Versorgungsforschung. Zwischen Routinedaten, Qualitätssicherung und Patientenorientierung. Siegburg: Asgard Verlagsservice 2015; 237-256.

8. Bowling A. Measuring disease: a review of diseasespecific quality of life measurement scales. Buckingham: Open University Press 1995.

9. Downs SH, Black N. The feasibility of creating a checklist for the assessment of the methodological quality both of randomized and non-randomised studies of health care interventions. J Epidemiol Community Health 1998; 52: 377-384.

10. Dwan K, Altman DG, Arnaiz JA et al. Systematic review of the empirical evidence of study publication bias and outcome reporting bias. PLoS ONE 2008; 3: e3081.

11. Icks A, Chernyak N, Bestehorn K et al Methoden der gesundheitsökonomischen Evaluation in der Versorgungsforschung. Gesundheitswesen 2010, 72: 917-933.

12. Swart E, Ihle P, Gothe H. Routinedaten im Gesundheitswesen: Handbuch Sekundärdatenanalyse: Grundlagen, Methoden und Perspektiven. 2. Aufl. Bern: Hans Huber 2014.

13. Deutsches Institut für Medizinische Dokumentation und Information (Dimdi). https://www. dimdi.de/static/de/klassi/icd-10-gm/index.htm Zugriff 04.07.2018

14. Kirsten N, Zander N, Gensel F et al. Populationbased epidemiologic study in venous diseases in Germany: Prevalence, comorbidity, and medical needs in a cohort of 19,104 workers. Project Final Report. University of Hamburg, 2016 and oral presentation at the Annual Meeting of the Euro- 
pean Wound Management Association (EWMA), Amsterdam 03.03.2017.

15. Statistisches Bundesamt (Destatis). https://www. destatis.de/DE/ZahlenFakten/GesellschaftStaat/ Gesundheit/Krankenhaeuser/Methoden/Krankenhausstatistik.html

16. Heyer K. Versorgungsepidemiologie des Ulcus cruris in Deutschland - Erkrankungshäufigkeit, Versorgungsqualität und Prädiktoren der Wundheilung. Wiesbaden: Springer 2016.

17. Rabe E, Pannier-Fischer F, Bromen K et al. Bonner Venenstudie der Deutschen Gesellschaft für Phlebologie - Epidemiologische Untersuchung zur Frage der Häufigkeit und Ausprägung von chronischen Venenkrankheiten in der städtischen und ländlichen Wohnbevölkerung. Phlebologie 2003; 32: 1-14.

18. Sommer R, Kiedrowski R, Stavermann T et al. Stellenwert und Umfang der phlebologischen Versorgung in dermatologischen Praxen. Phlebologie 2018. doi: $10.12687 /$ phleb2441-5-2018

19. Augustin M, Debus ES, Bruning G et al. Development and Validation of a Short Version of the Freiburg Life Quality Assessment for Chronic Venous Disease (FLQA-VS-10). Wound Medicine 2015; 8: 31-35.
20. Zschocke I, Bross F, Maier K et al. Quality of Life in Different Stages of Chronic Venous Insufficiency and Leg Ulcer. Dermatol Psychosom 2002; 3: 126-131.

21. Augustin M, Dieterle W, Zschocke I et al. Development and Validation of a Disease Specific Questionnaire on the Quality of Life of Patients with Chronic Venous Insufficiency. Vasa 1997; 26: 291-301.

22. Sommer R, Augustin M, Blome C. Erfassung von Lebensqualität und Verwendung patientenberichteter Endpunkte in der Praxis. Phlebologie 2017; 46: 334-339.

23. Purwins S, Herberger K, Debus ES et al. Cost-ofillness of chronic leg ulcers in Germany. Int Wound J 2010; 7: 7-102.

24. Augustin M, Brocatti LK, Rustenbach SJ et al. Cost-of-illness of leg ulcers in the community. 2014; 11: 283-92.

25. Kemper C, Sauer K, Glaeske G. Barmer GEK Heilund Hilfsmittelreport 2011. Auswertungsergebnisse der Barmer GEK Heil- und Hilfsmitteldaten aus den Jahren 2009 bis 2010. Schriftenreihe zur Gesundheitsanalyse, Band 10, 2011.

26. Heyer K, Augustin M. Therapie chronischer Wunden - Schwerpunkt Ulcus cruris. In: Sauer K,
Rothgang H, Glaeske G. Barmer GEK Heil- und Hilfsmittelreport 2014 - Schriftenreihe zur Gesundheitsanalyse, Band 28. Berlin: Asgard Verlagsservice GmbH 2014: 85-106.

27. Heyer K, Protz K, Glaeske G, Augustin M. Epidemiology and Use of Compression Treatment in Venous Leg Ulcers: Nationwide Claims Data Analysis in Germany. Int Wound J 2016; 14 (2): 338-343.

28. Augustin M, Rustenbach SJ, Debus S et al. Quality of Care in Chronic Leg Ulcer in the Community: Introduction of Quality Indicators and a Scoring System. Dermatology 2011; 222: 321-329.

29. Protz K, Heyer K, Doerler M et al. Compression therapy: Scientific Background and Practical Applications. J Dtsch Dermatol Ges 2014; 12: 794-802.

30. Heyer K, Protz K, Augustin M. Compression Therapy - Cross-sectional Observational Survey About Knowledge and Practical Treatment of Specialised and Non-specialised Nurses and Therapists. Int Wound J 2017; 14: 1148-1153.

31. Korn P, Patel ST, Heller JA et al. Why insurers should reimburse for compression stockings in patients with chronic venous stasis. J Vasc Surg 2002; 35: 950-957. 